[0212-7199 (2005) 22: 1: pp 28-30] ANALES DE MEDICINA INTERNA Copyright (C) 2005 ARAN EDICIONES, S.L.

AN. MED. INTERNA (Madrid) Vol. 22, N. ${ }^{\circ} 1$, pp. 28-30, 2005

\title{
Rotura parcial bilateral aquílea asociada a levofloxacino
}

\author{
F. L. LADO LADO, C. RODRÍGUEZ MORENO'1, M. VELASCO GONZÁLEZ, \\ C. DURÁN PARRONDO'², B. MOAR CALVO
}

Servicios de Medicina Interna, ${ }^{\prime}$ Farmacología Clínica. ${ }^{2}$ Atención Primaria de Lalín. Departamento de Medicina. Hospital Clínico Universitario. Santiago de Compostela. A Coruña

\author{
PARTIAL BILATERAL RUPTURE OF THE ACHILLES TENDON \\ ASSOCIATED TO LEVOFLOXACIN
}

\section{RESUMEN}

La tendinitis y rotura de tendón es un efecto indeseado bien conocido para fluoroquinolonas que también puede producirse con las nuevos agentes de este grupo. Aunque no existen muchos casos publicados para levofloxacino, esto es probablemente debido a lo reciente de su expansión. Aunque la descripción de casos no es el mejor método de valoración de la frecuencia de este problema, puede servir para alertar a los clínicos sobre la importancia de este efecto grave cuya frecuencia consideramos que ha sido infravalorada. En esta nota clínica presentamos un caso de rotura bilateral parcial del tendón aquíleo, probablemente asociado a levofloxacino.

PALABRAS CLAVE: Rotura tendón. Reacción adversa. Fluorquinolonas. Levofloxacino.

\begin{abstract}
Fluoroquinolones-associated tendonitis and tendon rupture are well described in the literature but these are not frequently observed and related to the new agents of this group, as levofloxacin. This is probably due to the recent introduction and expansion. Although epidemiological studies are needed to know the frequency of that levofloxacin-induced tendinopathies, case-report could alert to the physicians about this possible severe adverse reaction. We present a case of bilateral Achiles tendonitis with partial spontaneous rupture probably associated to levofloxacin.
\end{abstract}

KEY WORDS: Tendon rupture. Adverse reaction. Fluorquinolones. Levofloxacin.

Lado Lado FL, Rodríguez, Moreno C, Velasco González, M, Durán Parrondo C, Moar Calvo B. Rotura parcial bilateral aquílea asociada a levofloxacino. An Med Interna (Madrid) 2005; 22: 28-30.

\section{INTRODUCCIÓN}

La tendinitis y rotura de tendón es un efecto indeseado bien conocido para fluoroquinolonas que también puede producirse con los nuevos agentes de este grupo. Aunque la comunicación de casos y series de casos no es el método adecuado para valorar la frecuencia real de este problema, si permite eliminar esta percepción de efecto indeseado "excepcional" y alertar al clínico sobre su existencia e impacto sanitario. Este cambio de percepción es actualmente de gran importancia pues la utilización de levofloxacino se ha generalizado de manera espectacular e incluso es considerada como tratamiento de elección de la neumonía comunitaria en el adulto sin factores de riesgo (tanto intra como extrahospitalariamente) en recientes protocolos consensuados por diversas sociedades científicas de nuestro país (1). Por estas razones hemos considerado pertinente comunicar a esta revista el caso de rotura aquilea bilateral en relación con levofloxaciono que, recientemente, hemos tenido la oportunidad de diagnosticar.

\section{CASO APORTADO}

Varón de 79 años de $80 \mathrm{~kg}$ de peso que entre sus antecedentes presentaba enfermedad pulmonar obstructiva crónica, fibrilación auricular y epilepsia sensitivo-motora por lo que estaba a tratamiento desde años atrás con inhaladores de salmeterol, propionato de fluticasona y bromuro de ipratropio, acenocumarol según controles hematológicos, digoxina $0,25 \mathrm{mg} 5$ días/semana, furosemida 40 $\mathrm{mg} /$ día y gabapentina $900 \mathrm{mg} / \mathrm{día}$. El paciente se mantenía clínicamente estable hasta que ingresó en nuestro centro el día 4-03-04 por un aumento de su tos y disnea habituales con expectoración blanquecina de una semana de evolución sin otra sintomatología acompañante. En la exploración física destacaba taquipnea ( $28 \mathrm{rpm})$, auscultación cardiaca arrítmica, disminución del murmullo vesicular con roncus y sibilancias dispersas y edemas distales que dejaban fóvea.

\footnotetext{
Trabajo aceptado: 30 de julio de 2004
} 
En la analítica presentaba hipoxemia $\left(\mathrm{pO}_{2} 49 \mathrm{mmHg}\right)$ e hipercapnea $\left(\mathrm{pCO}_{2} 53 \mathrm{mmHg}\right)$, leucocitosis $\left(19.270 / \mathrm{mm}^{3}\right)$ con neutrofilia $(93 \%)$, urea $105 \mathrm{mg} / \mathrm{dl}$ (N: 12-44) y actividad de protrombina de 13\%. El resto de la hematimetría, coagulación, parámetros bioquímicos y el análisis elemental de orina fueron normales. Se realizaron diversos estudios microbiológicos que incluyeron cultivo y detección de BAAR en esputo que fueron negativos. En las pruebas de imagen, concretamente en la radiografía de tórax, se evidenciaron enfermedad quístico-cavitaria en lóbulo superior derecho e imágenes sugestivas de bronquiectasias de pared gruesa. En el electrocardiograma se registraron fibrilación auricular con frecuencia ventricular media de $100 \mathrm{lpm}$ y datos de hipertrofia ventricular izquierda. Además de mantener su tratamiento de base, el 04-03-04 se inició tratamiento con oxígenoterapia, tanda de corticoides en pauta descendente con metilprednisolona a partir de $60 \mathrm{mg} /$ día y, ante la sospecha clínica de infección respiratoria aguda que descompensaba su situación previa, levofloxacino a dosis de $500 \mathrm{mg}$ /día (los 5 primeros días endovenoso y 5 días más por vía oral) con lo que se observó una mejoría clínica evidente incluyendo desaparición de los edemas. El día 10-03-04 el paciente comenzó a quejarse de dolor de tobillos y dificultad para la deambulación presentando en ambos tumefacción y extravasación sanguínea. Ante la ausencia de antecedentes de traumatismo y con exploración sugestiva de rotura de tendón de Aquiles, se realizó una ecografía que evidenció rotura en tercio medio de ambos tendones de Aquiles que afectaba a gran parte de su espesor a dicho nivel. Ante la sospecha de su relación con la administración de levofloxacino, se procedió a su retirada el día 14-03-04, y también se realizó determinación de factor reumatoideo, anticuerpos antinucleares y HLA-B27 que fueron negativos. Se consultó con el Servicio de Traumatología que descartó inicialmente intervención quirúrgica y se decidió tratamiento conservador evolucionando en este sentido con mejoría progresiva de forma lenta pero significativa con mejoría del dolor y de los signos inflamatorios. El enfermo fue dado de alta el día 27-03-04.

\section{DISCUSIÓN}

Siguiendo el proceso habitual y necesario para establecer una relación de causalidad entre un efecto indeseado y el medicamento sospechoso (2) (algoritmo de Karch y Lasagna) exponemos que la rotura aquilea bilateral que presentó nuestro paciente cumple los requisitos exigidos:

1. Presenta una secuencia temporal compatible con la administración de levofloxacino ya que lo mas frecuente es que la tendinitis aparezca tras la primera semana de tratamiento y la rotura dentro de semana siguiente (dos desde el comienzo) (3-5). A pesar de ello es conveniente señalar que este problema puede producirse tan pronto como al día siguiente (6) y hasta 3 meses después de comenzado (y retirado) el tratamiento $(7,8)$.

2, Fueron cuidadosamente excluidas otras causas posibles, fundamentalmente traumatismos, artritis reumatoide y lupus eritematoso sistémico, aunque no podemos excluir una impli- cación concomitante de otros factores que pueden debilitar el tendón como son la edad avanzada y la toma de corticoides.

3. Se produjo una mejoría lenta pero apreciable de los síntomas al retirar el medicamento. Es necesario tener en cuenta que la recuperación de esta lesión es lenta y en ocasiones no completa, sobre todo si la rotura ha sido total. Así, en la serie de 100 casos de tendinitis con 31 roturas comunicada por Pierfitte y cols. (5) hubo una evolución favorable en el $75 \%$ de los casos de tendinitis y en el $48 \%$ de las roturas con un tiempo de recuperación que osciló entre 4-180 días siendo lo mas frecuente entre 30-60 días.

4. Este efecto ha sido previamente descrito y es bien conocido para las quinolonas en general $(5,9-20)$ y para levofloxacino en particular. De hecho coincidimos con Gómez Rodríguez y cols. (3), en que el problema puede ser mas frecuente de lo que hasta ahora se ha pensado. En el caso del levofloxacino, desde el primer caso comunicado por Lewis y cols. (21) ha habido varias publicaciones de casos $(3,4,22-24)$ y series de casos (7), y creemos que es razonable pensar que puede aparecer muchas mas pues la extensión de la utilización de este medicamento ha sido relativamente reciente. Estudios en animales apuntan a que el riesgo de afectación de tendones y cartílagos sería mayor para levofloxacino, ofloxacino y pefloxacino que para norfloxacino, ciprofloxacino y enoxacino debido a las diferencias que tienen en común ambos grupos en su estructura química (9).

Por estas razones y, ante la mas que evidente imposibilidad ética de realizar una reexposición de prueba, por estar absolutamente contraindicadas de por vida la nueva administración de quinolonas (ya que, aunque el mecanismo no está totalmente aclarado parece existir un fuerte componente de susceptibilidad individual), consideramos que éste se trata de un caso de rotura bilateral parcial del tendón aquíleo probablemente asociada a levofloxacino sin poder descartar ni afirmar el papel favorecedor de la metilprednisolona.

Por último queremos llamar la atención de los lectores sobre algunos puntos que consideramos relevantes: Este problema se ha observado con mayor frecuencia en hombres (razón 3:1), en mayores de 50 años con una media de 63 años, en coexistencia con fracaso renal y en asociación a corticoides $(4,5,25)$. Por tanto consideramos razonable pensar que éstas y cualquier otra situación que pueda aumentar la fragilidad tendinosa debe obligarnos a ser cautos en la utilización de quinolonas. También es importante recordar que la rotura puede aparecer muy rápidamente después de comenzado el tratamiento (6) pero que se han descrito casos incluso después de terminado el tratamiento (7) por lo que ante toda patología tendinosa de etiología no evidente, aconsejamos anamnesis farmacológica dirigida por medicamentos y síntomas para excluir la toma de quinolonas hasta un periodo de 3 meses atrás y retirada inmediata de la quinolona.

\section{Bibliografía}

1. Sociedad Española de Neumología y Cirugía Torácica (SEPAR), Sociedad Española de Quimioterapia (SEQ), Sociedad Española de Medicina Interna (SEMI), Sociedad Española de Medicina de Urgencias y Emergencias (SEMES). Tratamiento antibiótico empírico inicial de la neumonía adquirida en la comunidad en el paciente adulto inmunocompetente. Rev Esp Quimioterap 2003; 16: 457-66.

2. Karch FE, Lasagna L. Toward the operational identification of adverse drug reactions. Clin Pharmacol Ther 1977; 21: 247-54.

3. Gómez Rodríguez N, Ibáñez Ruán J, González Pérez M. Tendonitis aquílea bilateral y levofloxacino. An Med Interna (Madrid) 2004; 21: 154.

4. Kowatari K, Nakashima K, Ono A, Yoshihara M, Amano M, Toh S. Levofloxacin-induced bilateral Achilles tendon rupture: a case report 
and review of the literature. J Orthop Sci 2004; 9: 186-90.

5. Pierfitte C, Gillet P, Royer RJ. More on fluoroquinolone antibiotics and tendon rupture. N Engl J Med 1995; 332: 193.

6. Le-Huec JC, Schaeverbeke T, Chauveaux D, Rivel J, Dhais J, Le Rebeller A. Epicondylitis after treatment with fluroquinolone antibiotics. J Bone Joint Surg Br 1995; 77: 293-5.

7. Claudio Aros E, Claudio Flores W, Sergio Mezzano A. Tendinitis aquiliana asociada al uso de levofloxacino: comunicación de cuatro casos. Rev Med Chil 200; 130: 12177-81.

8. Stahlmann R, Lode H. Fluoroquinolones in the elderly: safety considerations. Drug Aging 2003; 20: 289-302.

9. Dukes MNG, Aronson JK. Meyler's side effects of drugs. Fourteenth edition. 2000; 852-6.

10. Van der Linden PD, Van de Lei J, Nab HW, et al. Achilles tendinitis associated with fluroquinolones. Br J Clin Pharmacol 1999; 48: 433-7.

11. Bailey RR, Kirk JA, Peddie BA. Norfloxacin induced rheumatic disease. N Z Med J 1983; 96: 590.

12. McGarvey WC, Singh D, Trevino SG. Partial Achilles tendon ruptures associated with fluoroquinolone antibiotics: a case report and literature review. Foot Ankle Int 1996; 17: 496-8.

13. Meyboom RH, Olsson S, Knol A, et al. Achilles tendonitis induced by pefloxacin and othe fluoroquinolone derivatives. Pharmacoepidemiol Drug Saf 1994; 3: 185-9.

14. Movin T, Gad A, Guntner P, et al. Pathology of the Achilles tendon in association with ciprofloxacin treatment. Foot Ankle Int 1997; 18: 297 9.

15. Pierfitte C, Royer RJ. Tendon disorders with fluoroquinolones. Therapie 1996; 51: 419-20.
16. Ribard P, Audisio F, Kahn MF, et al. Seven Achilles tendonitis including 3 complicated by rupture during fluoroquinolone therapy. J Rheumatol 1992; 19: 1479-81.

17. Royer RJ, Pierfitte C. Netter P. Features of tendon disorders with fluoroquinolones. Therapie 1994; 49: 75-6.

18. West MB, Gow P. Ciprofloxacin, bilateral Achilles tendonitis and unilateral tendon rupture: a case report. N Z Med J 1998; 111: 18-9.

19. Zabraniecki L, Negrier I, Vergne P, et al. Fluoroquinolone induced tendinopathy: report of 6 cases. J Rheumatol 1996; 23: 516-20.

20. Leone R, Venegoni M, Motola D, Moretti U, Piazzetta V, Cocci A et al. Adverse drug reactions related to the use of fluoroquinolone antimicrobials. An analysis of spontaneous reports and fluoroquinolone consumption data from three Italian regions. Drug Saf 2003; 26: 10920.

21. Lewis JR, Gums JG, Dickensheets DL. Levofloxacin-induced bilateral Achilles tendonitis. Ann Pharmacother 1999; 33: 792-5.

22. De la Red G, Mejía JC, Cervera R, Llado A, Mensa J, Font J. Bilateral Achilles tendinitis with spontaneous rupture induced by levofloxacin in a patient with systemic sclerosis. Clin Rheumatol 2003; 22: 367-8.

23. Casado Burgos E, Viñas Ponce G, Lauzurica Valdemoros R, Olivé Marqués A. Tendinitis por levofloxacino. Med Clin (Barc) 2000; 8: 319.

24. Nuno FJ, Noval J, Suárez M, Guinea O. Dolor aquíleo e impotencia funcional en paciente con enfermedad pulmonar obstructiva crónica con neumonía. Rotura tendinosa por levofloxacino. Rev Clin Esp 2001; 201: 539-40.

25. Hurst M, Lamb H, Scott L, Figgitt D. Levofloxacin. An updated review of its use in the treatment of bacterial infections. Drugs 2002; 62: 2127 67. 